\title{
Separate Valuation Subsystems for Delay and Effort Decision Costs
}

\author{
Charlotte Prévost, ${ }^{1,2}$ Mathias Pessiglione, ${ }^{3}$ Elise Météreau, ${ }^{1,2}$ Marie-Laure Cléry-Melin, ${ }^{2,3}$ and Jean-Claude Dreher ${ }^{1,2}$ \\ ${ }^{1}$ Cognitive Neuroscience Center, Centre National de la Recherche Scientifique, Unité mixte de recherche 5229, Reward and Decision Making Team, 69675 \\ Bron, France, ${ }^{2}$ Université Claude Bernard Lyon 1, 69003 Lyon, France, and ${ }^{3}$ Institut du Cerveau et de la Moëlle épinière, INSERM Unité mixte de recherche \\ en Santé 975, Motivation, Brain, and Behavior Team, Hôpital Pitié-Salpêtrière, 75013 Paris, France
}

Decision making consists of choosing among available options on the basis of a valuation of their potential costs and benefits. Most theoretical models of decision making in behavioral economics, psychology, and computer science propose that the desirability of outcomes expected from alternative options can be quantified by utility functions. These utility functions allow a decision maker to assign subjective values to each option under consideration by weighting the likely benefits and costs resulting from an action and to select the one with the highest subjective value. Here, we used model-based neuroimaging to test whether the human brain uses separate valuation systems for rewards (erotic stimuli) associated with different types of costs, namely, delay and effort. We show that humans devalue rewards associated with physical effort in a strikingly similar fashion to those they devalue that are associated with delays, and that a single computational model derived from economics theory can account for the behavior observed in both delay discounting and effort discounting. However, our neuroimaging data reveal that the human brain uses distinct valuation subsystems for different types of costs, reflecting in opposite fashion delayed reward and future energetic expenses. The ventral striatum and the ventromedial prefrontal cortex represent the increasing subjective value of delayed rewards, whereas a distinct network, composed of the anterior cingulate cortex and the anterior insula, represent the decreasing value of the effortful option, coding the expected expense of energy. Together, these data demonstrate that the valuation processes underlying different types of costs can be fractionated at the cerebral level.

\section{Introduction}

Decision making can be seen as a process of maximizing utilities associated with different options. A domain in which mathematical formalization of utility function has proven particularly efficient in describing choice behavior is delay discounting. "Delay discounting" refers to the empirical finding that both humans and animals value immediate rewards more than delayed rewards. A large number of behavioral studies have demonstrated that the subjective value of a delayed reward may be discounted hyperbolically (Ainslie, 1975; Frederick et al., 2002). Similarly, because effort carries a cost, a reward may carry a higher value if it is easily obtained than if it is obtained through great effort. Yet, it is still unclear whether humans devalue rewards associated with physical effort in a fashion similar to that with which they devalue those associated with delays.

Standard theories of economic decision making do not distinguish between decisions related to different types of costs, such as delay or effort costs (Kahneman and Tversky, 1979). A choice is made after a valuation stage, regardless of the nature of the cost.

Received May 31, 2010; revised June 28, 2010; accepted Aug. 13, 2010.

This work was funded by a Marie Curie International reintegration grant and a Fyssen Foundation grant to J.-C.D. Many thanks to the staff of the Centre de Neuroimagerie de Recherche (K. Nigaud, R. Valabregue, E. Barbinet, and E. Bertasi), J. Redouté, and R. Neveu for helpful assistance.

Correspondence should be addressed to Jean-Claude Dreher, Centre National de la Recherche Scientifique, Unité mixte de recherche 5229, Reward and Decision-Making Team, Cognitive Neuroscience Center, 67 Boulevard Pinel, 69675 Bron, France. E-mail: dreher@isc.cnrs.fr.

DOI:10.1523/JNEUROSCI.2752-10.2010

Copyright $\odot 2010$ the authors $\quad 0270-6474 / 10 / 3014080-11 \$ 15.00 / 0$
However, lesion studies in rodents suggest at least partial dissociations between the neural structures used to assess delay- and effort-based decision making (Rudebeck et al., 2006; Walton et al., 2006; Floresco et al., 2008). Despite the fundamental importance of these animal studies for paving the way in identifying the neural substrates involved in making decisions about delay and effort costs, it is unknown whether these circuits can be generalized to humans and whether they specifically concern the valuation stage. Indeed, specifying the roles of brain structures specifically involved during the valuation stage, and not during the subsequent waiting/effort periods, has proven difficult because animal studies cannot pinpoint exactly at what point in the decision-making process a lesioned animal is impaired. Yet, a number of them have shown that it is neither the ability to wait nor the exertion of effort, per se, that is impaired by the use of control conditions (Rudebeck et al., 2006).

Here, using model-based functional neuroimaging, we sought to extend the framework of subjective utility functions to the domain of effort discounting, allowing us to assess whether the human brain computes subjective values related to rewards associated with different types of costs in distinct sets of brain structures. We designed similar delay- and effort-discounting paradigms using erotic pictures as rewards, involving passive delay periods in the second range and real physical effort using a hand grip. On each trial, subjects made a choice based on an incentive cue (fuzzy erotic image) between a variable costly option (waiting or exerting more effort), leading to viewing the erotic picture in clear for a "long" time (large reward), and a 

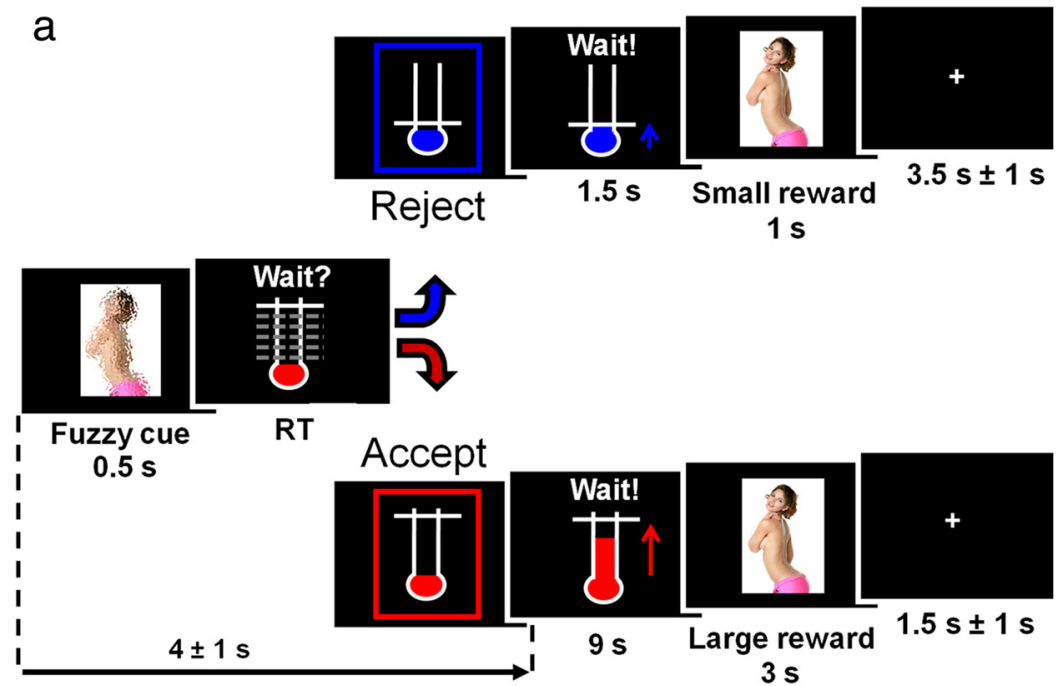

b
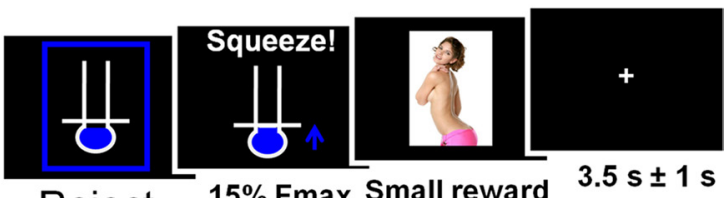

Reject

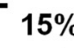

$+1.5 \mathrm{~s}$

$1 \mathrm{~s}$
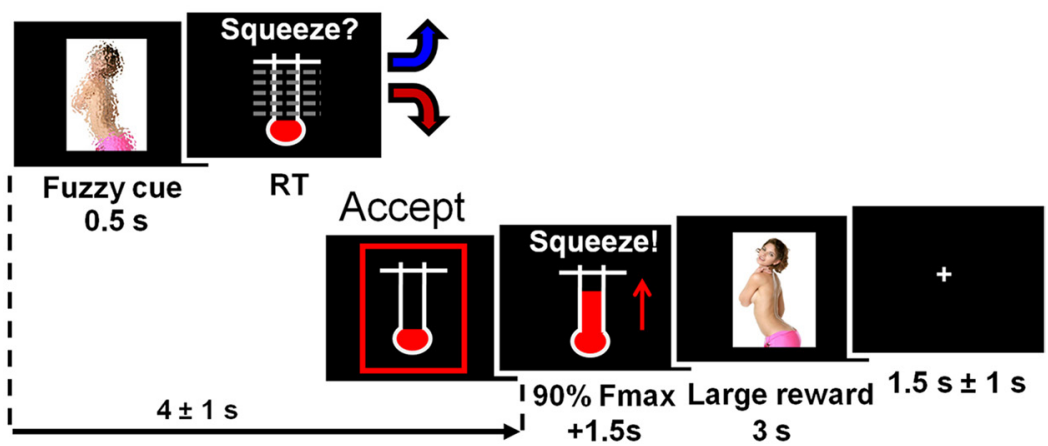

$+1.5 \mathrm{~s}$

Figure 1. $\quad \boldsymbol{a}, \boldsymbol{b}$, Delay-discounting ( $\boldsymbol{a}$ ) and effort-discounting $(\boldsymbol{b})$ paradigms using primary rewards. On each trial, a fuzzy erotic picture briefly appeared on a screen and was followed by the instruction "Wait?" or "Squeeze?," together with a thermometer indicating one of 6 possible levels of the proposed delay period to wait or effort to invest (ranging from 1.5 to $9 \mathrm{~s}$ for the delay, and from 15 to $90 \%$ of subjects' maximal strength for the effort). Depending on the incentive cue and the proposed level of cost, subjects chose between the costly option and a default option having a minimal cost (1.5 s of waiting or $15 \%$ of maximal strength to exert). Then, they either waited passively during the delay period or produced the effort, before seeing the erotic picture clearly for a short time period (small reward) if they rejected the costly option, or a longer period of time (large reward) if they accepted it. The outcome and the intertrial interval lasted for a total of 4.5 s plus a jitter of $\pm 1 \mathrm{~s}$ in both options, avoiding that subjects adopted the strategy of choosing more the default option to see more pictures.

default option, having a minimal cost but leading to viewing the picture in clear for a "short" time (small reward) (Fig. 1).

We used primary rewards rather than secondary rewards such as money because monetary rewards neither allow subjects to experience the reward at the time of delivery inside the scanner nor allow them to experience the delay period, often ranging from days to months (McClure et al., 2004; Kable and Glimcher, 2007; Wittmann et al., 2007; Ballard and Knutson, 2009). Moreover, the subjective rating of each fuzzy erotic picture was used to compute subjective values without assuming that subjective value increases linearly with the objective amount of money (Kable and Glimcher, 2007; Pine et al., 2009).

\section{Materials and Methods}

Participants. Eighteen young, healthy, heterosexual men (mean age: $23.1 \pm 1.8$ years), participated in the study. All the participants were males because men are generally more responsive to visual sexual stimuli compared with women, both in terms of behavioral arousal and brain response (Hamann et al., 2004; Sescousse et al., 2010), and to avoid potential influence of the menstrual cycle known to have an effect on reward processing in women (Dreher et al., 2007). Two participants initially enrolled were excluded from data analysis because of inappropriate calibration of the task for the preferences of these subjects. The study was approved by the Paris Pitié-Salpêtrière Hospital ethics committee, and written informed consent was obtained from all subjects. All participants were right-handed, as assessed by the Edinburgh Handedness Questionnaire (mean score: $0.83 \pm 0.17$ ) and were screened by a psychiatrist for history of psychiatric or neurological illness, as assessed by the Mini-International Neuropsychiatric Interview and the Hospital Anxiety and Depression Scale (HAD) (mean HAD score: $3.32 \pm 1.46$ ). None of the subjects showed impulsivity patterns, as assessed by the Barratt impulsiveness scale (Patton et al., 1995) (mean score: $53.78 \pm$ 6.09). Subjects' sexual orientation was assessed using the French analysis of sexual behavior questionnaire (Spira et al., 1993) and sexual arousability was measured with the Sexual Arousability Inventory (SAI) (Hoon and Chambless, 1998) (mean score SAI: $92.76 \pm$ 12.34), which ensured that subjects showed a "standard" sexual arousability. Subjects were asked to avoid any sexual relationship for $24 \mathrm{~h}$ before the scanning session.

Delay/effort discounting task. Subjects were first asked to read the instructions of the task. To ensure that subjects understood the task and to familiarize them with the hand grip, they were trained on a practice version outside the scanner room with fuzzy cues and clear outcome pictures that were different from those subsequently used in the scanner (to avoid any habituation effect). During training and inside the scanner, subjects' maximal strength was measured using a magnetic resonance imaging (MRI)-compatible handgrip (designed by Eric Featherstone, Wellcome Trust Centre for Neuroimaging, London, UK). They were then taken inside the scanner and were invited to find an optimal body position, while lying down with the power grip in their right hand, the arm resting over the belly. The power grip was made up of two molded plastic cylinders, which compressed an air tube when squeezed. The tube led to the control room, where it was connected to a transducer capable of converting air pressure into voltage. Thus, compression of the two cylinders by an isometric handgrip resulted in the generation of a differential voltage signal, linearly proportional to the force exerted. The signal was fed to the stimuli presentation computer (PC) via a signal conditioner (CED 1401, Cambridge Electronic Design). The task was programmed on a PC using the matlab toolbox Cogent 2000 (http://www.vislab.ucl.ac.uk/cogent_2000.php).

There were five sessions (lasting $\sim 9 \mathrm{~min}$ ) composed of 48 trials each, leading to a total of 240 trials. The behavioral task was composed of the following two conditions: a delay condition and an effort condition, which were presented in a random order. Each trial started with the presentation of a cue $(0.5 \mathrm{~s})$ showing an erotic fuzzy picture of a naked woman. The screen then displayed the instruction "Wait?" or "Squeeze?," together with a thermometer indicating a proposed level that 
could be displayed at six different heights (Fig. 1). This level indicated either the proposed delay period, which could last between 1.5 and $9 \mathrm{~s}$ (increment: $1.5 \mathrm{~s}$ ) or the proposed force to exert, varying between 15 and $90 \%$ (increment: 15\%) of the subject's maximal strength. In the delay condition, subjects had to decide whether they chose the costly option (i.e., wait the longer delay period indicated by the height of the level on the thermometer) to see the cue clearly for $3 \mathrm{~s}$ (large reward), or whether they chose the default option (i.e., wait for a fixed short period of time of $1.5 \mathrm{~s}$ ) to view the picture for $1 \mathrm{~s}$ (small reward). Similarly, in the effort condition subjects decided whether it was worth investing in a stronger effort to see the picture clearly for $3 \mathrm{~s}$ or simply to invest in a small effort corresponding to $15 \%$ of their maximal strength to see the cue for $1 \mathrm{~s}$. Subjects made their choice with a response pad in their left hand (accept costly option, forefinger; reject costly option, middle finger).

After their choice, the thermometer was framed in a red/blue rectangle to visually indicate which option was chosen. Then, subjects were involved in waiting or in exerting an effort proportional to the level indicated on the thermometer. In the delay condition, subjects passively waited until the required time had elapsed and the thermometer was filled up to the indicated level on the thermometer. In the effort condition, subjects squeezed the hand grip until they reach the indicated level on the thermometer. Finally, at the outcome, according to their choice (costly vs default option), they viewed the erotic picture clearly for 3 or $1 \mathrm{~s}$. The duration of the display of the cue plus the proposition (instruction screen) was $4 \mathrm{~s}$ plus a jitter of $\pm 1000 \mathrm{~ms}$. If the subject did not make his decision during this time, the trial was aborted and the instruction "Pay attention" was displayed for $2 \mathrm{~s}$.

The trial ended with an intertrial interval of $1.5 \mathrm{~s}$ plus a jitter of $\pm 1 \mathrm{~s}$ when subjects accepted the proposition and $3.5 \mathrm{~s}$ plus a jitter of $\pm 1 \mathrm{~s}$ when they rejected it. In this way, the outcome and the intertrial interval lasted for a total of $4.5 \mathrm{~s}$ plus a jitter of $\pm 1 \mathrm{~s}$ in both options, to avoid that subjects adopt the strategy of choosing more the default option to see more pictures. Note that we chose not to make each trial the same length overall because this would have considerably extended the duration of the experiment (each delay trial would have lasted $17.5 \mathrm{~s}$ on average) and because this may have led subjects to no longer accept as many trials as is the case in the current version of the experiment, since short delayed options would lead to waiting overall the exact same duration as the delayed rewarded option. Subjects were explicitly asked to make their choices according to both the fuzzy cue and the proposed level of delay/ effort and to weigh the cost and benefit of each option. They were also told that systematically choosing the less costly option would not allow them to see more pictures or finish the experiment earlier and that the sexual intensity of the fuzzy pictures was not linked to the level of proposed delay/effort since these images were presented in a random order. The six different levels were randomly presented across sessions with an average of 20 trials per level and per condition.

Stimuli. Each picture was presented twice: once with a fuzzy appearance at the beginning of each trial (incentive cue) and once in a clear form at the outcome. Erotic pictures of women were used becausecontrary to monetary rewards-they allowed us to include waiting periods in the second range and to have subjects really experience the reward delivery in each trial during scanning. Despite their critical sociobiological importance, erotic stimuli have not been studied as reinforcers, but rather as arousing stimuli in passive viewing paradigms focusing on sexual function.

Five hundred fifty erotic pictures were selected from the World Wide Web according to two objective criteria: women had to be alone and their face visible. These pictures underwent a glass effect in Adobe Photoshop 7.0 using the following parameters: distortion, 10; smoothness, 5; texture type, frosted; scaling, 100; and without invert texture.

The use of fuzzy cues had several advantages. First, displaying them at the beginning of each trial allowed preserving the saliency of the clear picture displayed at the outcome phase and avoided the habituation that could have occurred if only the clear pictures had been repeated (Agmo, 1999). Second, they had the power to motivate the subject by giving an idea of the content of the picture without unveiling the rewarding aspect of the clear picture. Hence, they allowed us to partially guide subjects' choices. Moreover, performing postscan ratings of each fuzzy cue, fur- ther used in subjective value computation, allowed us to avoid the assumption that the subjective value of monetary rewards increases linearly with an objective amount of money (Pine et al., 2009).

Five hundred sixteen of these pictures were rated by 30 men in a pilot experiment using the software Presentation 9.9 (Neurobehavioral Systems) (the other 34 pictures were used as examples). The 240 best rated pictures considered as the most rewarding pictures were selected for our delay/effort discounting task. The rationale for using different viewing durations for the clear picture was based on the fact that subjects work harder to see an attractive face longer because it is more rewarding (Hayden et al., 2007).

The duration of the erotic pictures seen clearly and the various delays and efforts used were initially piloted in several behavioral experiments, including $\sim 50$ participants, to ensure that an approximately equal number of immediate and delayed options were chosen by the subjects.

Ratings of the fuzzy cues. At the end of the scanning session, subjects were asked to rate the 240 fuzzy cues they saw during the experiment. They had to answer the question: "How much would you like to see this fuzzy picture in clear?" by moving a cursor along a scale going from 1 ("I do not want to see the fuzzy picture in clear at all") to 9 ("I extremely want to see the fuzzy picture in clear"') with an increment of 0.2 . This incentive measure of each picture was used to compute the subjective values (corresponding to the reward intensity, called $A$ in Eq. 1 below). The ratings of the cues were also used in behavioral analysis (see Fig. 2) and in functional MRI (fMRI) region of interest (ROI) analyses (see below, Computational model, and Figs. 5b, 6b), after normalizing and sorting them into one of four categories (category 1 being the lowest rated pictures and category 4 being the highest rated pictures). For the latter analyses, we collapsed the original nine levels of ratings into four bins to ensure a sufficient number of repetitions in each bin and to generate robust statistics. Note that the levels of delays and effort presented on the thermometer were not rated after the scan and were assumed to be perceived in a linear fashion by the subjects.

Computational model. We used a hyperbolic function to compute the subjective values of delayed rewards because it has previously been shown to offer the best account of delay discounting in both humans and animals (Ainslie, 1975; Frederick et al., 2002). A similar hyperbolic function was used to compute the subjective values of rewards associated with a larger effort. On a trial-by-trial basis, the model estimated the subjective value of the reward associated with the costly option and the default option in the delay condition $\left(\mathrm{SV}_{\mathrm{D}}\right)$ and in the effort condition $\left(\mathrm{SV}_{\mathrm{E}}\right)$. These subjective values were computed as follows:

$$
\begin{aligned}
& \mathrm{SV}_{\mathrm{D}}=A_{\mathrm{D}} * x_{\mathrm{D}} /\left(1+C_{\mathrm{D}} * k_{\mathrm{D}}\right) \\
& \mathrm{SV}_{\mathrm{E}}=A_{\mathrm{E}} * x_{\mathrm{E}} /\left(1+C_{\mathrm{E}} * k_{\mathrm{E}}\right)
\end{aligned}
$$

Here, $A$ corresponds to the reward intensity (ratings of the fuzzy cues), $x$ is a subject-specific constant corresponding to the ratio between viewing the clear picture for $3 \mathrm{~s}$ (large reward) and viewing it for $1 \mathrm{~s}$ (small reward), $C$ corresponds to the proposed level of the cost, and $k$ is a subject-specific constant corresponding to the discount factor. This model was then used to create a parametric regressor corresponding to the estimated subjective value of the rewards associated with the costly option in a given condition for analysis of brain images.

The associated probability (or likelihood) of choosing the costly option was estimated by implementing the softmax rule, as follows:

$$
\begin{aligned}
& P(\text { delayed option })=1 /\left(1+\exp \left(-\mathrm{SV}_{\mathrm{D}} / \beta_{\mathrm{D}}\right)\right) \\
& P(\text { effortful option })=1 /\left(1+\exp \left(-\mathrm{SV}_{\mathrm{E}} / \beta_{\mathrm{E}}\right)\right)
\end{aligned}
$$

This standard stochastic decision rule allowed us to compute the probability of choosing the costly option according to its associated subjective value (supplemental Figs. 4, 5, available at www.jneurosci.org as supplemental material). The temperature $\beta$ is a free parameter concerning the randomness of decision making. The parameters $x, k$, and $\beta$ were adjusted using the least square method to minimize the distance between the behavioral choice and the probability of choice estimated by the model, across all sessions and subjects. 
Table 1. Foci of activity (from GLM1) showing correlation with the subjective value of costly rewards

\begin{tabular}{|c|c|c|c|c|c|}
\hline \multirow[b]{2}{*}{ Brain regions } & \multirow[b]{2}{*}{ Hemisphere } & \multicolumn{3}{|c|}{ MNI peak coordinates } & \multirow[b]{2}{*}{$t$ values } \\
\hline & & $x$ & y & $z$ & \\
\hline \multicolumn{6}{|c|}{ Positive correlation with subjective value of delayed rewards } \\
\hline Ventral striatum & Right & 10 & 10 & -12 & 6.51 \\
\hline Putamen & Left & -20 & 4 & 12 & 5.47 \\
\hline Ventromedial prefrontal cortex & Left & -10 & 24 & -12 & 5.83 \\
\hline Lateral PFC & Left & -34 & 34 & 8 & 5.79 \\
\hline Middle frontal gyrus & Left & -22 & -20 & 62 & 6.04 \\
\hline \multirow[t]{2}{*}{ Precentral gyrus } & Left & -58 & -18 & 38 & 5.95 \\
\hline & Right & 24 & -26 & 46 & 5.55 \\
\hline Inferior parietal lobule & Left & -42 & -34 & 30 & 6.03 \\
\hline Thalamus & Right & 14 & -18 & 4 & 6.03 \\
\hline Postcentral gyrus & Right & 48 & -20 & 34 & 5.19 \\
\hline \multirow[t]{2}{*}{ Occipital gyrus } & Left & -26 & -100 & 4 & 4.58 \\
\hline & Right & 34 & -98 & -6 & 5.78 \\
\hline \multicolumn{6}{|c|}{ Positive correlation with subjective value of effortful rewards } \\
\hline Precentral gyrus & Left & -36 & -18 & 66 & 4.29 \\
\hline Postcentral gyrus & Left & -34 & -28 & 50 & 4.19 \\
\hline \multicolumn{6}{|c|}{ Negative correlation with subjective value of effortful rewards } \\
\hline Anterior cingulate cortex & Right & 6 & 24 & 28 & 6.01 \\
\hline \multirow[t]{2}{*}{ Anterior insula } & Left & -30 & 22 & 10 & 5.35 \\
\hline & Right & 30 & 26 & 8 & 4.97 \\
\hline Inferior parietal lobule & Right & -68 & -36 & 24 & 4.91 \\
\hline Superior temporal gyrus & Right & 58 & -42 & 16 & 4.86 \\
\hline \multirow[t]{2}{*}{ Occipital cortex } & Left & -2 & -86 & -10 & 4.62 \\
\hline & Right & 14 & -76 & 10 & 4.54 \\
\hline
\end{tabular}

All reported foci survived a voxel-level threshold of $p<0.001$, uncorrected for multiple comparisons and a cluster-level threshold of $p<0.05$ corrected for multiple comparisons. MNI, Montreal Neurological Institute.

fMRI data acquisition. Imaging was performed on a 3 tesla TRIO TIM scanner (Siemens Medical Solutions). T2*-weighted echo-planar images (EPIs) were acquired in an interleaved order with blood oxygendependent level (BOLD) contrast. Whole-brain functional images were acquired in 35 slices $(128 \times 128$ voxels, $2 \mathrm{~mm}$ slice thickness, $2 \mathrm{~mm}$ interslice gap, $30^{\circ}$ off of the anterior commissure-posterior commissure line at a repetition time of $1.98 \mathrm{~s}$ ). We used a tilted plane acquisition sequence to optimize functional sensitivity in the orbitofrontal cortex (Weiskopf et al., 2006). T1-weighted structural images were also acquired, coregistered with the mean EPI, segmented, and normalized to a standard T1 template. EPI images were analyzed in an event-related manner, within a general linear model (GLM), using the statistical parametric mapping software SPM5 (Wellcome Department of Imaging Neuroscience, London, UK). The first three volumes of each session were discarded to allow for T1 equilibration effects. Before the analysis, the images were corrected for slice time artifacts, spatially realigned, normalized using the same transformation as structural images, and spatially smoothed with an $8 \mathrm{~mm}$ full-width at half maximum Gaussian kernel.

GLM1: main FMRI data statistical analysis. We used one main linear regression model to account for our data (GLM1). Each trial was modeled as having three different phases, corresponding to the decisionmaking phase, the cost-enduring phase and the outcome phase. Trials were sorted according to the condition (delay or effort) and distributed into separate regressors. Two regressors were used to account for the decision phase: one for the delay condition and one for the effort condition. The subjective value of the reward associated with the costly option was included as a parametric modulation on these two regressors. Two regressors were used to account for the experienced delay period and the effort investment period (one for each condition). Two additional regressors accounted for the outcome phase, one for the small reward and the other one for the large reward, regardless of the condition. Therefore, the design matrix contained eight regressors of interest, all convolved with a canonical hemodynamic response function (HRF) and modeled with boxcars having the durations of the corresponding event. To correct for motion artifact, subject-specific realignment parameters were modeled as covariates of no interest. Linear contrasts of regression coefficients were computed at the individual subject level and then taken to a group level random-effects analysis (one-sample $t$ test).

We applied a threshold of $p<0.001$ (uncorrected) with a clusterbased threshold level of $p<0.05$ corrected for multiple comparisons. All the results concerning this model exclusively concern the decisionmaking phase.

Additional control analyses: GLM2 and GLM3. Because the subjective value reflects a ratio between the rating of the cue and the level of proposed delay or effort, an increased BOLD response with subjective value of the costly reward could reflect a positive correlation with the ratings of the incentive, a negative correlation with the proposed level of delay or effort, or a combination of both the rating and the level of the cost. Conversely, a decreased BOLD response with subjective value of the costly reward could reflect a negative correlation with the ratings of the incentive, a positive correlation with the proposed level of delay or effort, or a combination of both the rating and the level of the cost. To investigate whether the brain regions showing activity correlating with subjective value of the costly reward were better accounted by the subjective values (Eqs. 1 and 2) than by the rating of the incentive and the level of the cost in a given condition at the time of choice, we performed two additional fMRI analyses (GLM2 and GLM3) with these single parameters as parametric regressors (rating of the cue alone and level of the cost alone modeled in separate regressors for the delay and effort conditions), assessing whether both the strength (peak $z$-score) and spatial extent (cluster size in millimeters) of the activity of the cluster correlating with subjective value were larger than those of these single parameters (supplemental Fig. 7, supplemental Tables 1-3, available at www.jneurosci. org as supplemental material). These two analyses were similar to our main GLM1 except that the subjective value regressor was replaced by a single regressor containing the ratings of the fuzzy cue in the first analysis (GLM2) and the level of the cost in the second one (GLM3).

ROI analyses. To gain more insight into the correlational analysis obtained with subjective value, we performed additional ROI analyses with the parameters of the task (rating and level of cost) to plot the respective influences of the proposed level of effort or delay and the influence of the ratings of the incentive in different brain regions. Regions of interest, conducted with the extension of SPM MarsBaR (http://marsbar.sourceforge.net/), were defined functionally from the initial whole-brain analysis (GLM1). Each ROI was created by taking the intersection of the functional cluster of interest and an 8-mm-radius sphere centered on the highest peak voxel of the cluster corresponding to the Montreal Neurological Institute coordinates reported in Table 1. In the delay condition, the functional clusters of interest were the ventromedial prefrontal cortex (vmPFC) and the ventral striatum because of 
their reported role in coding the subjective value of delayed rewards in the monetary domain (Tanaka et al., 2004; Kable and Glimcher, 2007; Peters and Büchel, 2009). In the effort condition, for the positive correlation with subjective value of the effortful reward, the only ROI was the primary motor cortex (M1) because it was the main activation found in this regression analysis. For the negative correlation with subjective value of the effortful reward, ROIs were defined in the anterior cingulate cortex (ACC) and anterior insula cortex because a previous study in rodents reported effects of ACC lesions on effort-related decision making (Rudebeck et al., 2008), because higher ACC and anterior insula activities were reported during anticipation of higher reward in the context of effortbased cost-benefit valuation in humans (Croxson et al., 2009) and because previous fMRI studies also reported an important role for the ACC-anterior insula network when making decisions under uncertainty (Huettel et al., 2005; Grinband et al., 2006). Figures $3 b$ and $4 b$ show the parameter estimates obtained from GLM1, GLM2, and GLM3 in each ROI. Moreover, to illustrate the activities correlating with subjective value of the high reward in the delay and the effort condition, the $\beta$ estimates of $\mathrm{SV}_{\mathrm{D}}$ and $\mathrm{SV}_{\mathrm{E}}$ were plotted (Figs. $3 c, 4 c$ ). Note that no statistical test was performed on these SVs because the ROI analysis is not independent from the whole-brain analysis.

To further illustrate the shape of the correlational analysis with the subjective value, the rating, and the level of proposed cost, we estimated three additional GLMs (GLM4, GLM5, and GLM6). This allowed us to extract and isolate the percent signal change (averaged across subjects) according to the subjective value of the reward associated with the costly option (GLM4), the rating of the incentive cue (GLM5), and the levels of delay and effort costs (GLM6) (see Figs. 5, 6). In GLM4, each trial was modeled as having three different phases, corresponding to the decisionmaking phase, the cost-enduring phase, and the outcome phase. Trials were sorted according to the condition (delay/effort). Twelve regressors were used to account for the subjective values at the time of the decision: 6 for the delay condition and 6 for the effort condition. Two regressors were used to account for the experienced delay period and the effort investment period (one for each condition). Two additional regressors accounted for the outcome phase, one for the small reward and the other one for the large reward. Therefore, the design matrix contained 16 regressors of interest, all convolved with a canonical HRF. To correct for motion artifacts, subject-specific realignment parameters were modeled as covariates. For each session of each subject, the subjective values were equally divided in these six categories, from the lowest sixth to the highest sixth (being the high category). This allowed us to extract the percent signal change for each of these categories, which was averaged across sessions for each subject and then averaged across all subjects (Figs. $5 a$ and $6 a$ ). To show the effects of the incentive value of the cue (ratings) and the level of proposed delay (costs) in the brain regions correlating positively with the subjective value of the delayed option, we performed GLM5 and GLM6. GLM5 had the same three different phases, with the four categories of rating of the cue as parametric modulation at the time of choice instead of the six categories of subjective values (Figs. $5 b$ and $6 b$ ). The other GLM (GLM6) performed to plot the graphs of percentage BOLD change as a function of the levels of costs also had the same three different phases, with the six levels of proposed costs as parametric modulation at the time of choice instead of the four categories of rating of the cue (Figs. $5 c, 6 c$ ).

Cost-enduring phase and outcome phase. Finally, we took advantage of our fMRI design, allowing us to distinguish the brain regions involved in the valuation processes concomitant with the decision from the brain regions recruited during the cost-enduring phase and those activated by the large versus small rewards in each of the two conditions. For this analysis, we used a similar model to our main GLM1, except that this model included a parametric modulation by the level of the cost during the delay period and during the effort exerted, and also separated the large and small rewards for the delay and effort conditions. These functional data were analyzed by constructing a set of boxcars having the duration of each corresponding event (i.e., decision phase lasting the response times (RTs), delay/effort phase lasting the delay effectively waited and the effort exerted, and outcome phase lasting $1 \mathrm{~s}$ for the small reward and $3 \mathrm{~s}$ for the large reward), all convolved with a canonical hemodynamic response function. For the anticipation contrasts, we used a threshold of $p<0.001$, uncorrected with a cluster-level threshold of $p<$ 0.05 corrected for multiple comparisons. For the outcome contrasts, we used a threshold of $p<0.005$, uncorrected, because of our very specific focus on ventromedial prefrontal cortex and striatum, which have both been robustly implicated in responding to the receipt of reward in a large number of studies (Knutson et al., 2001; Smith et al., 2010).

Finally, in a last analysis, we investigated whether the activation observed at the time of the rewarded outcome was modulated by the level of delay or effort that had just been experienced. We used a similar model to our main GLM1, except that this model included a parametric modulation by the level of the cost during the delay period and during the effort exerted, and also during the outcome period for the delay and effort conditions separately. These functional data were analyzed by constructing a set of boxcars having the duration of each corresponding event (i.e., decision phase lasting the RTs, delay/effort phase lasting the delay effectively waited and the effort exerted, and outcome phase lasting $1 \mathrm{~s}$ for the small reward and $3 \mathrm{~s}$ for the large reward), all convolved with a canonical hemodynamic response function. For this last analysis, we used a threshold of $p<0.001$, uncorrected with a cluster-level threshold of $p<0.05$ corrected for multiple comparisons.

\section{Results}

\section{Behavioral results}

Subjects provided clear evidence of both delay and effort discounting in their preferences, choosing more frequently the costly option for lower proposed levels of delay $\left(F_{(5,75)}=46.30\right.$; $p<0.001)$ and effort $\left(F_{(5,75)}=54.67 ; p<0.001\right.$ ) (Fig. 2a,c), as well as for higher ratings of the incentive (assessed by postscan ratings of the fuzzy cues) in both the delay $\left(F_{(3,45)}=7.21 ; p<\right.$ $0.001)$ and the effort conditions $\left(F_{(3,45)}=14.71 ; p<0.001\right)$ (Fig. $2 b, d)$. Interactions between the level of the cost and ratings of the incentive were observed in both the delay $\left(F_{(15,25)}=1.84, p<\right.$ $0.05)$ and effort $\left(F_{(15,25)}=2.68, p<0.001\right)$ conditions, because of the fact that increasing ratings of the fuzzy cue more strongly influenced the choice toward the costly option at intermediate levels of delay/effort (supplemental Figs. 1, 2, available at www. jneurosci.org as supplemental material).

Response times increased with the proposed level of delay $\left(F_{(5,75)}=4.14 ; p<0.01\right)$ or effort $\left(F_{(5,75)}=6.46 ; p<0.001\right)$, indicating subjects' reluctance to wait longer or to engage in more effort (supplemental Fig. 3, available at www.jneurosci.org as supplemental material). These results show that subjects integrated in their decision both the benefit associated with the cue and the cost indicated by the proposed level of delay/effort. An alternative interpretation of our RTs findings in terms of difficulty of the decision is not supported by our data because it would have predicted significantly higher RTs for the level closest to the point of subjective equivalence between the two options (where subjects are equally likely to choose the costly or noncostly options), compared with RTs obtained for other levels. This is not what was observed since RTs for a delay of $6 \mathrm{~s}$ (point of subjective equivalence in the delay condition) did not significantly differ from RTs of $4.5,7.5$, and $9 \mathrm{~s}$ (all $p>0.3$, paired $t$ tests). Similarly, RTs for level of 70\% maximal strength (point of subjective equivalence in the effort condition) did not significantly differ from RTs in the 45, 60,75, and 90\% of maximal strength conditions (all $p>0.1$, paired $t$ tests).

Figure 2, $e$ and $f$, show the subjective value of rewards associated with the costly and noncostly options in both conditions, estimated by hyperbolic functions depending on the rating of the cue and the level of the cost (see Materials and Methods, Eqs. 1, 2; and supplemental Figs. 4, 5, available at www.jneurosci.org as supplemental material). As expected, the subjective value of the 

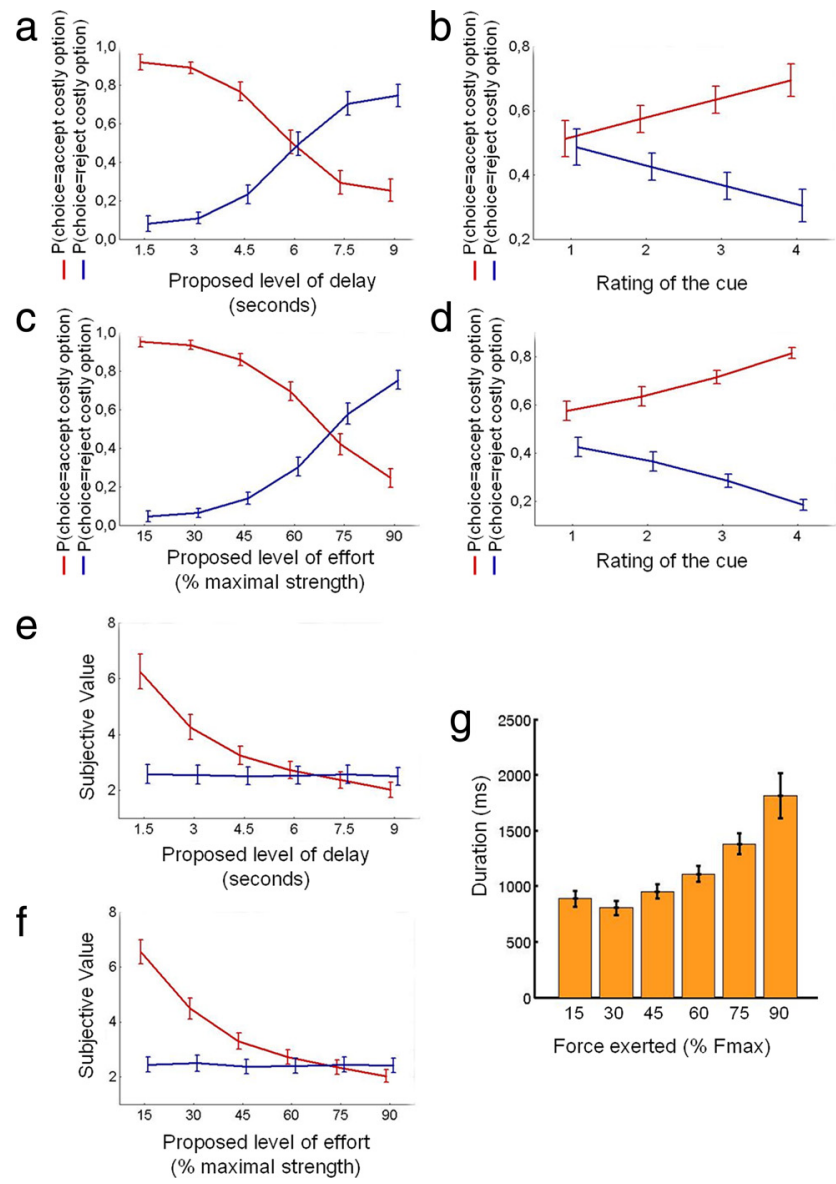

Figure 2. Behavioral results. $\boldsymbol{a}, \boldsymbol{c}$, Probability of choosing the costly option according to the proposed level of delay $(\boldsymbol{a})$ or effort $(\boldsymbol{c})$. The red/blue lines represent averaged ratios of accepted/rejected trials across subjects, for each level of proposed delay or effort. $\boldsymbol{b}, \boldsymbol{d}$, Probability of choosing the costly option according to the rating of the fuzzy cue in the delay $(\boldsymbol{b})$ and effort $(\boldsymbol{d})$ conditions. The red/blue lines represent averaged ratios of accepted/rejected trials across subjects, for each category of incentive value of the cue. $\boldsymbol{e}, \boldsymbol{f}$, Subjective value of rewards associated with the two available options according to the proposed level of delay $(\boldsymbol{e})$ and effort $(\boldsymbol{f})$. The $\mathrm{red} / \mathrm{blue}$ lines represent the subjective value of the reward associated with the costly/default option. Error bars represent SEM. $\boldsymbol{g}$, Time required to exert each level of effort in the effortenduring phase averaged for each level of effort across sessions and subjects.

reward associated with the costly option decreased as the associated proposed level of delay or effort increased, demonstrating that delay and effort were effectively perceived as costs. This result also demonstrates, to the best of our knowledge for the first time, that the subjective value of a high reward associated with a larger effort is discounted hyperbolically (see also below, Model comparison), as previously demonstrated for the subjective value of delayed reward. Moreover, our experimental design was effective to have subjects devalue primary rewards in a few seconds.

Finally, we investigated how much time was needed to exert each of the six levels of effort (Fig. $2 g$ ). The time difference between exerting the largest effort compared with the smallest effort was shorter than a second, and the time required to exert all the efforts lasted $<2 \mathrm{~s}$, and were all significantly $<3$ s (i.e., the time corresponding to the second smallest level of proposed delay) $(p<0.001)$. Thus, the time required to exert the different efforts should not be considered as an issue in our analysis, showing that valuation of effort and delay (occurring during the decision phase) are dissociable in the brain. a

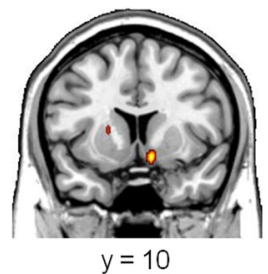

b
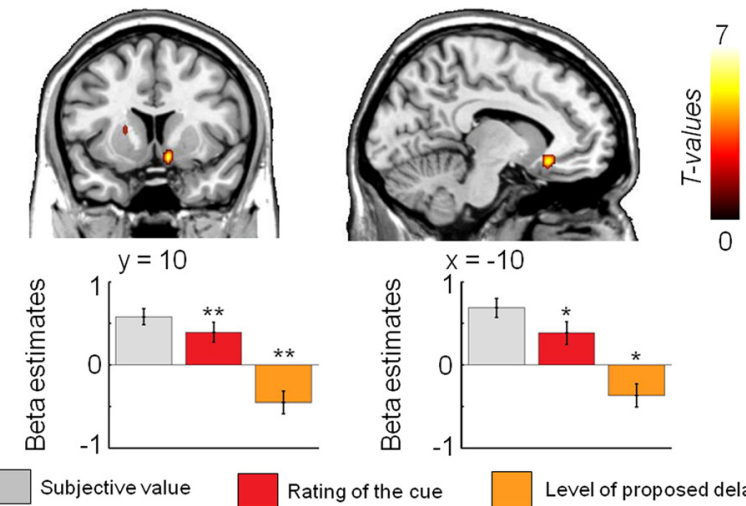

C

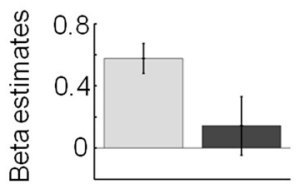

Subjective value of the delayed reward

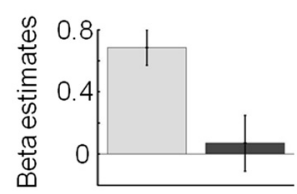

Subjective value of the effortful reward

Figure 3. Results from the parametric regression analysis showing areas in which activity is positively correlated with the subjective value of delayed rewards (random-effects group analysis: $p<0.001$, uncorrected; $p<0.05$ cluster corrected). $\boldsymbol{a}$, Activity in the ventral striatum and ventromedial prefrontal cortex increases as the subjective value of delayed rewards increases (GLM1). $\boldsymbol{b}$, Plots of the $\beta$ values representing the slope of the linear regression between neural activity and the subjective value of the delayed reward (light gray, GLM1), the rating of the cue (red, GLM2), and the proposed level of delay (orange, GLM3) in each ROI. ${ }^{*} p<0.05,{ }^{* *} p<$ 0.01 , one-sample $t$ tests comparing the effect sizes to zero. Note that no statistics were performed in the ROI concerning the plot of the $\beta$ estimate of the subjective value of the delayed reward, because such analysis would not be independent of the whole-brain analysis. The gray bar plot is simply shown to illustrate the results presented in the regression analysis with subjective value. c, Plots of the $\beta$ values representing the slope derived from the linear regression between the subjective value of the costly reward and neural activity in each ROl in both the delay (light gray) and the effort condition (dark gray) (GLM1).

\section{Model comparison}

The behavioral fits of the probability of choosing the costly options are shown in supplemental Figures 4 and 5 (available at www.jneurosci.org as supplemental material) for each subject. To further ensure that a hyperbolic function was a better fit to subjects' behavior, we compared hyperbolic and exponential fits (Schweighofer et al., 2006). The distance $\Delta$ between the modeled and observed data, estimated by the least-square method, revealed that the hyperbolic fit was better than the exponential fit to explain subjects' behavior $(p<0.001)$. Moreover, the hyperbolic function fitted equally well subjects' behavior in the delay and effort conditions because the distance $\Delta$ between the hyperbolic fit and the behavioral data did not differ between these two conditions $\left(\Delta_{\text {delay }}=0.1393 \pm\right.$ $\left.0.049, \Delta_{\text {effort }}=0.1412 \pm 0.063 ; p=0.93\right)$.

\section{fMRI results}

Subjective valuation of delayed/effortful erotic rewards

We used parametric regression analyses to identify the brain regions showing activity correlating with the subjective value of the reward associated with the costly option in each condition during the decision-making phase. First, we found that activity in the ventral striatum and vmPFC increases with higher subjective value of delayed rewards (Fig. $3 a$, Table 1). Additional fMRI analyses (see Materials and Methods) confirmed that the activity of these two brain regions was better accounted by the subjective value (GLM1) than by the rating of the incentive (GLM2) or the level of delay (GLM3) taken independently (Fig. 3b, Table 1; 
supplemental Fig. $7 a$, supplemental Table 1 , available at www.jneurosci.org as supplemental material). Other brain regions, such as the lateral prefrontal cortex, also showed increasing activities with higher subjective value of delayed rewards (Table 1), but this activity was better explained by the rating alone than by the subjective value of the delayed reward, both in terms of strength (peak $z$-score) and spatial extent (cluster size) (supplemental Fig. 7a, supplemental Table 1, available at www.jneurosci. org as supplemental material). No brain region showed activity negatively correlating with subjective value in the delay condition ( $p>0.005$, uncorrected).

When searching for brain regions showing activity correlating with the subjective value of the high reward associated with a more substantial effort, a positive correlation was found only in the left primary motor (M1) cortex (Fig. 4a, Table 1), contralateral to the right hand squeezing the grip. However, this $\mathrm{M} 1$ activity was better explained by the rating of the cue (GLM2) than by the increasing subjective value of the reward associated with a larger effort (GLM1) (supplemental Fig. $7 b$, supplemental Table 2 , available at www.jneurosci.org as supplemental material), suggesting that it reflects preparation to engage in the subsequent effort with higher incentive value of the fuzzy cue, rather than a valuation signal. Moreover, increased left M1 activity was also observed when more effort had to be exerted in the cost-enduring phase, showing that the same brain region is involved in motor preparation and in execution of the action (supplemental Fig. $6 b$, available at www. jneurosci.org as supplemental material).

In contrast, a negative correlation with the subjective value of the large reward associated with a more substantial effort was found in the ACC-insula network (Fig. 4b, Table 1). This activity reflects that when the proposed effort cost increases, the effortful reward is devalued. Further fMRI analyses showed that activity of the ACC was better accounted by the subjective value of the effortful option than by the rating of the incentive (GLM2) and the effort level (GLM3), whereas the anterior insula activity was better explained by the proposed level of effort (GLM3) than by other parameters (Fig. $4 c$; supplemental Fig. $7 c$, supplemental Table 3, available at www.jneurosci.org as supplemental material). Thus, the ACC may be in a particular position to integrate both the benefit associated with the fuzzy cue and the effort cost while the anterior insula assesses the level of energy expenditure required to reach a proposed effort.

\section{ROI analyses}

ROI analyses were conducted for the vmPFC and ventral striatum in the delay condition and for the ACC and bilateral insula in the effort condition. Categorical analyses allowed us to extract the signal percentage change in these regions for different categories of subjective value (GLM4) (Figs. 5a, 6a), different categories of rating (GLM5) (Figs. 5b, 6b), and the different levels of proposed delay or effort (GLM6) (Figs. $5 c, 6 c$ ) distributed in separate regressors for delay and effort at the time of choice. Figures $5 c$ and $6 c$ show that activity of the ventral striatum/vmPFC and the ACC/anterior insula valuation systems code in opposite fashion delayed rewards and future energetic expenses: activity of the ACC/insula coding the decreasing subjective value of effortful rewards increase with larger proposed efforts whereas ventral striatal/vmPFC activity coding the increasing subjective value of delayed rewards show decreased activity with longer delays. This indicates that delay and effort costs engage distinct neural mechanisms.

\section{Separate systems for subjective valuation of delayed/effortful rewards}

To test whether the brain networks identified with subjective valuation of delay and devaluation of effort engage separate neural systems, we also performed direct comparisons of the activities of brain regions in which the positive correlation with subjective value of the delayed reward was significantly greater (respectively lower) than the negative correlation with subjective value of the effortful reward (supplemental Tables 4, 5, available at www.jneurosci.org as supplemental material). These direct whole-brain statistical comparisons of the effects of subjective value in the effort and delay conditions, demonstrated the specificity of the brain networks identified in the valuation of delayed reward and in the devaluation of effortful reward.

Finally, we performed two conjunction analyses, one searching for brain regions positively correlating with the subjective value of the costly reward during both the delay and effort conditions, and the other searching for regions positively correlating with the subjective value during the delay condition and nega- 
a

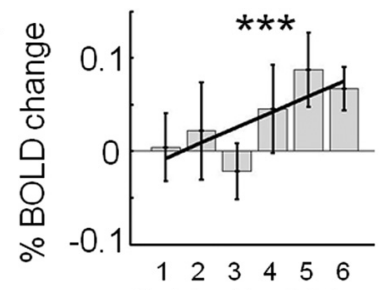

b

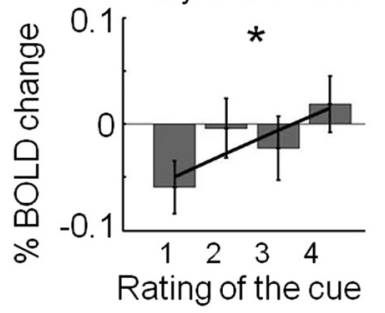

C

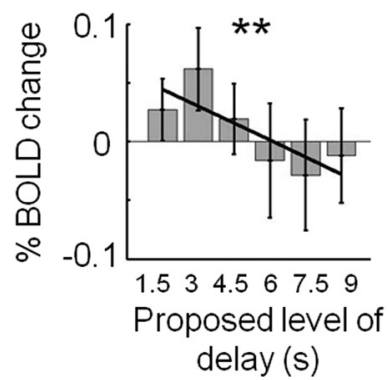

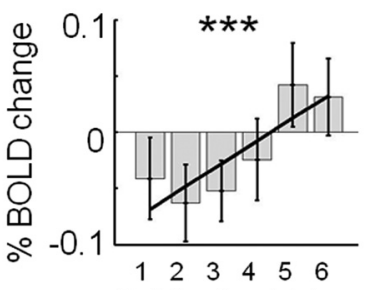

Subjective Value
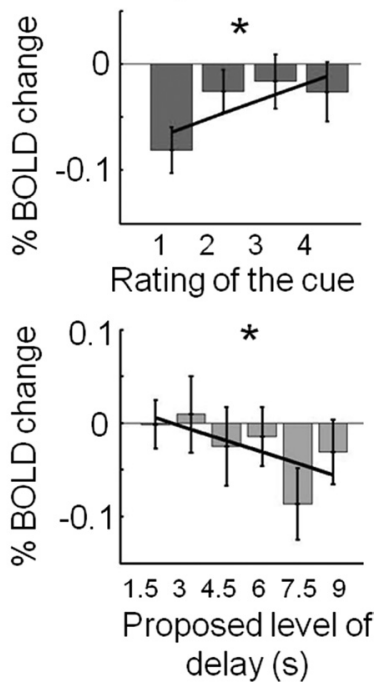

Figure 5. $\boldsymbol{a}-\boldsymbol{c}$, Percent signal change in ventral striatum (left column) and vmPFC (right column). $\boldsymbol{a}$, The subjective value of the delayed reward with trials binned into six categories such that each bin has an equal numbers of trials (GLM4). $\boldsymbol{b}$, The four categories of the incentive value of the cue (GLM5). c, The six proposed levels of delay cost (GLM6). Error bars indicate SEM. Linear regressions were performed to test the linear relationship between percentage BOLD change and subjective value $(\boldsymbol{a})$, cue ratings $(\boldsymbol{b})$, and proposed level of delay $(\boldsymbol{c}){ }^{*} p<0.05$, ${ }^{* *} p<0.01,{ }^{* * *} p<0.001$.

tively correlating with subjective value during the effort condition. No voxel survived in these conjunctions $(p>0.05$, cluster corrected at $p<0.05$ ), supporting the view that there is neither a common neural system tracking the value of both delayed and effortful rewards nor valuing delayed reward and devaluing effortful reward.

\section{Cost-enduring and outcome phases}

When investigating which brain regions were modulated by the level of cost that subjects experienced during the delay and effort periods, subsequent to the valuation stage, we found that waiting longer only activated the ACC sulcus (supplemental Fig. 6a, available at www.jneurosci.org as supplemental material, greenblue). In contrast, exerting more effort activated the adjacent ACC gyrus (supplemental Fig. $6 a$, available at www.jneurosci.org as supplemental material), the right amygdala, the dopaminergic midbrain, and the left motor cortex (supplemental Fig. $6 b$, available at www.jneurosci.org as supplemental material).

Finally, in each condition we investigated which brain regions were specifically more active at the outcome for the large reward (lasting $3 \mathrm{~s}$ ), following the experience of the cost (delay or effort), compared with the small reward (1 s). In the delay condition, we found that the large reward induced higher activity in the ventral caudate and the vmPFC compared with the small reward (supplemental Fig. $8 a$, available at www.jneurosci.org as supplemental material). Similarly, in the effort condition these same brain regions were also found to be more active for the large reward

relative to the small reward (supplemental Fig. $8 b$, available at www.jneurosci.org as supplemental material).

In a separate analysis, we found, in the delay condition, a positive correlation between the time waited and activity in the ventral striatum and ventromedial PFC at the time of outcome (supplemental Fig. $8 c$, available at www.jneurosci.org as supplemental material). There was no significant correlation between the effort exerted and BOLD response at the outcome in the effort condition.

\section{Discussion}

This study provides the first evidence that humans devalue rewards associated with physical effort (i.e., effort discounting) in a strikingly similar fashion to those they devalue that are associated with delays, as accounted for by the representation of subjective values used in revealed preference theories (Bernoulli, 1982; Gul and Pesendorfer, 2005). Our key novel findings are that, although behaviorally higher levels of delay and effort were effectively perceived as costly, at the neural level a striking dissociation was observed between mesolimbic regions (e.g., ventral striatum and vmPFC), showing a positive correlation with the subjective value of the delayed reward, and between the anterior cingulate cortex and the anterior insula, showing a negative correlation with the subjective value of effortful reward (therefore appearing to devalue rewards that require greater effort). Critically, although activity of the ventral striatum and vmPFC increased with higher incentive value of the subsequent reward (rating of fuzzy cue) and decreased as the imposed delay to a reward increased (Figs. $3 b$, $5 b, c)$, the ACC-anterior insula network, coding devaluation of the effortful reward (Fig. 4b), showed increasing (Fig. 6c)-rather than decreasing-activity with higher levels of proposed effort, supporting the hypothesis that the computations of the subjective value of effortful and delayed rewards involve fundamentally different neural mechanisms.

Thus, our neuroimaging data indicate that distinct valuation systems compute the subjective value of rewards associated with delay and effort costs and that these valuation subsystems code in opposite fashion delayed and effortful rewards, although similar behavioral choices and discount utility functions were observed for delay and effort costs. The fact that the valuation processes underlying decisions associated with different types of costs can be fractionated at the cerebral level is consistent with the existence of multiple independent and interacting functional systems in the brain (Rangel et al., 2008; Dreher, 2009), including neuroimaging demonstrations that distinct brain systems value delayed reward, risk, and probabilistic reward (Kuhnen and Knutson, 2005; Dreher et al., 2006; Preuschoff et al., 2006; Peters and Büchel, 2009). These different valuation systems may lead to diverging conclusions about the best option to choose if one had to decide between waiting for a long time or exerting a large effort to receive a high reward.

Our delay-discounting findings are in accordance with a number of previous works in animals (Cardinal et al., 2001) as well as with delay-discounting neuroimaging studies using monetary rewards in humans (Kable and Glimcher, 2007; Wittmann et al., 2007; Ballard and Knutson, 2009; Gregorios-Pippas et al., 2009). In particular, they are broadly consistent with a recent fMRI study revealing that the ventral striatum and anterior medial PFC track the subjective value of delayed monetary rewards (Kable and Glimcher, 2007). Although our vmPFC was more posterior than the anterior medial PFC reported in this study, it overlapped with the vmPFC region associated with valuation in other paradigms (Plassmann et al., 2007; Behrens et al., 2008; 
Hare et al., 2009). Lesions and pharmacological manipulation of the ventral striatum and orbitofrontal cortex of rodents induce behavioral deficits during delaybased paradigms (Cardinal et al., 2001; Mobini et al., 2002; Winstanley et al., 2004; Rudebeck et al., 2006), and increased activity of the ventral striatum has often been reported with expected value and its basic components, reward probability and magnitude (Knutson et al., 2005; Dreher et al., 2006; Yacubian et al., 2006; Kable and Glimcher, 2007; GregoriosPippas et al., 2009). Together, these findings suggest that subjective valuation signals of erotic rewards really experienced inside the scanner are computed in similar limbic frontostriatal networks than nonexperienced secondary (monetary) rewards, delayed from minutes to month/years. Therefore, the neural response to both primary and secondary reinforcers follows similar delay-discounting functions, suggesting that valuation of delayed rewards may obey common basic principles of neuronal computation, regardless of the reward nature and the delay duration incurred before reward delivery.

It is still unclear whether there are differences between the valuation of different types of primary rewards that are physiologically necessary (e.g., juice/water) or not (erotic pictures). In a previous study, an impatient " $\beta$ system," which includes the ventral striatum and the vmPFC, was preferentially activated by choices between an immediate reward and a delayed juice reward in intertemporal choices (McClure et al., 2007). These findings may appear contradictory with our current delay-discounting results using erotic pictures, because when the subjective value of the delayed reward increased, subjects were more likely to choose the option leading to the delayed reward. However, our findings are difficult to compare to this previous study because a different type of computational modeling approach was used to account for the data. According to these authors, there are two different valuation processes during intertemporal choice: the impatient $\beta$-process, steeply discounting all nonimmediate rewards; and a more patient $\delta$ process, active in both immediate and delayed trials, less steeply discounting all delayed rewards. In contrast, our study, which adopted the approach proposed by Kable and Glimcher (2007), suggests that a single valuation system encodes the hyperbolically discounted value of both delayed and immediate rewards. Moreover, our report that activity in the ventral striatum and vmPFC vary when only the delay to reward changes provides direct evidence that these regions do not exclusively value immediate rewards.

A very different pattern of results was observed in the effort condition. First, the left primary motor cortex was more activated with higher subjective value of the effortful reward (GLM1), but this activity was better explained by the rating of the cue (GLM2) (supplemental Fig. $7 b$, available at www.jneurosci.org as supplemental material), suggesting that it reflects preparation to engage in the subsequent effort with higher incentive value of the fuzzy cue, rather than a valuation signal. Consistent with this, M1 activity was also observed when exerting the effort (supplemental Fig. $6 b$, available at www.jneurosci.org as supplemental material), showing that the same brain region is actually involved in motor preparation and in execution of the action. More importantly, a negative correlation with the subjective value of the large reward associated with a more substantial effort was found in the ACC-anterior insula network. This decreasing activity reflects that when the proposed effort cost increases, the effortful reward is devalued. This devaluation reflects that the proposed effort is perceived in terms of engagement of energy since higher ACC/anterior insula activity was also observed with increasing proposed level of effort, before any effort is exerted (in contrast, no brain region showed increased activity with higher proposed delay). Yet, the ACC response was better accounted for by the subjective value of the effortful option than by the level of proposed effort or the rating of the cue, indicating that the ACC effectively encodes a value signal integrating effort and benefit, as opposed to a more basic motor preparatory signal.

These results demonstrate a critical role of the ACC-anterior insula network for evaluating whether or not it is worth producing a given effort for the reward at stake. Importantly, ACC activity did not correlate with the subjective value of the delayed reward. This implies that the ACC is not merely involved whenever it is necessary to evaluate two competing options but instead specifically when evaluating the benefits of exerting more effort for a higher reward compared with a less rewarding option that requires less energy expenditure. These findings may explain the 
impairment of ACC-lesioned animals in effort-based decision making (Walton et al., 2003; Rudebeck et al., 2006) as being the result of a dysfunction in evaluating the reward associated with the costly effort and additionally predict that anterior insula lesions in humans may lead to deficits in assessing effort expenses. A recent human $\mathrm{AMRI}$ study also provides evidence that the ACC codes the interaction between the expected reward amount and the effort cost (Croxson et al., 2009). However, in this study subjects did not decide between more or less effortful options. Overall, the present findings show that the ACC plays a pivotal role in evaluating whether the proposed level of effort is worth being engaged, considering the benefit of one course of action (Walton et al., 2006; Rushworth et al., 2007). Our ACC finding also converges with monkey electrophysiological recordings indicating that ACC neuronal activity reflects the integrated value of a course of action, encoding a combination of several decision variables, such as potential reward amount and effort cost (Kennerley et al., 2009) and with the fact that ACC neurons encode a monkey's progression through the series of work steps toward reward-although in this task effort and delay were not separated (Shidara and Richmond, 2002). However, our results are not a mere confirmation of animal studies suggesting that there are different brain systems for effort and delay costs. Indeed, these studies used focalized brain lesions that may affect the valuation, the decision, or the delay/effort execution stages. Our model-based fMRI approach allowed us to specifically investigate which brain structures showed activity positively or negatively correlating with the subjective value of effortful or delayed primary rewards. Another advantage of this approach is the ability to isolate this valuation stage from the delay period and effort exertion phases (supplemental Fig. 7, available at www.jneurosci.org as supplemental material).

The ventral striatum, the ACC, and the vmPFC are strongly implicated in cost/benefit decision making. Yet, their relative roles have never been directly simultaneously compared using a similar design for decisions concerning delay and effort costs. Our paradigm, which separately manipulates the benefit (cue) and the cost, indicates that during the effort condition ventral striatal and vmPFC responses did not correlate with the subjective value of the effortful reward or with the level of proposed effort. This result demonstrates that the ventral striatal value signal is not discounted by effort, and two recent rodent studies have come to a similar conclusion (Walton et al., 2009; Gan et al., 2010). In particular, ventral striatal phasic dopamine release has been reported to reflect the magnitude of the benefit, but not the expected effort (Gan et al., 2010). Consistent with this finding, ventral striatal activity positively correlated with the rating of the cue (benefit) in both the delay and effort conditions, but was not modulated by the proposed level of effort (supplemental Fig. 9, available at www.jneurosci.org as supplemental material). Although the relationship between striatal dopamine release and the BOLD signal is still unclear, analyses of variations in genes involved in striatal dopamine transmission establish a link between higher striatal BOLD signal and dopamine synaptic availability during reward processing (Dreher et al., 2009). A direct relationship between midbrain dopamine synthesis and BOLD signal changes in the reward system has also been demonstrated (Dreher et al., 2008). Together, our current results help to pinpoint the specific roles of brain regions specifically involved during the valuation stage of decisions related to delay and effort costs.

In summary, our data shed new light on value-based decisionmaking signals in the human brain by revealing that distinct val- uation subsystems are engaged for different types of costs, and code in opposite fashion delayed rewards and future energetic expenses. From an evolutionary perspective, separate valuation systems may have evolved through the need of responding to distinct types of costs in different environments. For example, some primate species are willing to tolerate delay costs but are less inclined to exert more effort and to travel farther to obtain greater reward, whereas the opposite is true for other species (Stevens et al., 2005). Finally, our demonstration that separate neural systems track the subjective value of rewards associated with different types of costs may prove useful for understanding impulsive (delay aversion) and apathetic (effort aversion) behavior in a number of neuropsychiatric disorders known to impair the capacity to select between available options based on an evaluation of their potential costs and benefits (Paulus, 2007).

\section{References}

Agmo A (1999) Sexual motivation-an inquiry into events determining the occurrence of sexual behavior. Behav Brain Res 105:129-150.

Ainslie G (1975) Specious reward: a behavioral theory of impulsiveness and impulse control. Psychol Bull 82:463-496.

Ballard K, Knutson B (2009) Dissociable neural representations of future reward magnitude and delay during temporal discounting. Neuroimage 45:143-150.

Behrens TE, Hunt LT, Woolrich MW, Rushworth MF (2008) Associative learning of social value. Nature 456:245-249.

Bernoulli D (1982) The works. Boston: Birkhauser.

Cardinal RN, Pennicott DR, Sugathapala CL, Robbins TW, Everitt BJ (2001) Impulsive choice induced in rats by lesions of the nucleus accumbens core. Science 292:2499-2501.

Croxson PL, Walton ME, O’Reilly JX, Behrens TE, Rushworth MF (2009) Effort-based cost-benefit valuation and the human brain. J Neurosci 29:4531-4541.

Dreher J-C (2009) Decomposing brain signals involved in value-based decision making. In: Handbook of reward and decision making (Dreher J-C, Tremblay L, eds), pp 137-164. New York: Academic, Elsevier.

Dreher JC, Kohn P, Berman KF (2006) Neural coding of distinct statistical properties of reward information in humans. Cereb Cortex 16:561-573.

Dreher JC, Schmidt PJ, Kohn P, Furman D, Rubinow D, Berman KF (2007) Menstrual cycle phase modulates reward-related neural function in women. Proc Natl Acad Sci U S A 104:2465-2470.

Dreher JC, Meyer-Lindenberg A, Kohn P, Berman KF (2008) Age-related changes in midbrain dopaminergic regulation of the human reward system. Proc Natl Acad Sci U S A 105:15106-15111.

Dreher JC, Kohn P, Kolachana B, Weinberger DR, Berman KF (2009) Variation in dopamine genes influences responsivity of the human reward system. Proc Natl Acad Sci U S A 106:617-622.

Floresco SB, Tse MT, Ghods-Sharifi S (2008) Dopaminergic and glutamatergic regulation of effort- and delay-based decision making. Neuropsychopharmacology 33:1966-1979.

Frederick S, Loewenstein G, O’Donoghue T (2002) Time discounting and time preference: a critical review. J Econ Lit 40:351-401.

Gan JO, Walton ME, Phillips PE (2010) Dissociable cost and benefit encoding of future rewards by mesolimbic dopamine. Nat Neurosci 13:25-27.

Gregorios-Pippas L, Tobler PN, Schultz W (2009) Short-term temporal discounting of reward value in human ventral striatum. J Neurophysiol 101:1507-1523.

Grinband J, Hirsch J, Ferrera VP (2006) A neural representation of categorization uncertainty in the human brain. Neuron 49:757-763.

Gul F, Pesendorfer W (2005) The case for mindless economics. Working paper. Princeton, NJ: Department of Economics, Princeton University.

Hamann S, Herman RA, Nolan CL, Wallen K (2004) Men and women differ in amygdala response to visual sexual stimuli. Nat Neurosci 7:411-416.

Hare TA, Camerer CF, Rangel A (2009) Self-control in decision-making involves modulation of the vmPFC valuation system. Science 324:646-648.

Hayden BY, Parikh PC, Deaner RO, Platt ML (2007) Economic principles motivating social attention in humans. Proc Biol Sci 274:1751-1756.

Hoon EF, Chambless D (1998) Sexual arousability inventory (SAI) and sexual arousability inventory-expanded (SAI-E). In: Handbook of sexuality- 
related measures (Davis CM, YWL, Bauserman R, Schreer G, Davis SL, eds), pp 71-74. Washington, DC: American Psychiatric Association.

Huettel SA, Song AW, McCarthy G (2005) Decisions under uncertainty: probabilistic context influences activation of prefrontal and parietal cortices. J Neurosci 25:3304-3311.

Kable JW, Glimcher PW (2007) The neural correlates of subjective value during intertemporal choice. Nat Neurosci 10:1625-1633.

Kahneman D, Tversky A (1979) Prospect theory: an analysis of decision under risk. Econometrica 47:263-291.

Kennerley SW, Dahmubed AF, Lara AH, Wallis JD (2009) Neurons in the frontal lobe encode the value of multiple decision variables. J Cogn Neurosci 21:1162-1178.

Knutson B, Fong GW, Adams CM, Varner JL, Hommer D (2001) Dissociation of reward anticipation and outcome with event-related fMRI. Neuroreport 12:3683-3687.

Knutson B, Taylor J, Kaufman M, Peterson R, Glover G (2005) Distributed neural representation of expected value. J Neurosci 25:4806-4812.

Kuhnen CM, Knutson B (2005) The neural basis of financial risk taking. Neuron 47:763-770.

McClure SM, Laibson DI, Loewenstein G, Cohen JD (2004) Separate neural systems value immediate and delayed monetary rewards. Science 306:503-507.

McClure SM, Ericson KM, Laibson DI, Loewenstein G, Cohen JD (2007) Time discounting for primary rewards. J Neurosci 27:5796-5804.

Mobini S, Body S, Ho MY, Bradshaw CM, Szabadi E, Deakin JF, Anderson IM (2002) Effects of lesions of the orbitofrontal cortex on sensitivity to delayed and probabilistic reinforcement. Psychopharmacology (Berl) 160:290-298

Patton JH, Stanford MS, Barratt ES (1995) Factor structure of the Barratt impulsiveness scale. J Clin Psychol 51:768-774.

Paulus MP (2007) Decision-making dysfunctions in psychiatry-altered homeostatic processing? Science 318:602-606.

Peters J, Büchel C (2009) Overlapping and distinct neural systems code for subjective value during intertemporal and risky decision making. J Neurosci 29:15727-15734.

Pine A, Seymour B, Roiser JP, Bossaerts P, Friston KJ, Curran HV, Dolan RJ (2009) Encoding of marginal utility across time in the human brain. J Neurosci 29:9575-9581.

Plassmann H, O’Doherty J, Rangel A (2007) Orbitofrontal cortex encodes willingness to pay in everyday economic transactions. J Neurosci 27:9984-9988.

Preuschoff K, Bossaerts P, Quartz SR (2006) Neural differentiation of expected reward and risk in human subcortical structures. Neuron 51:381-390.

Rangel A, Camerer C, Montague PR (2008) A framework for studying the neurobiology of value-based decision making. Nat Rev Neurosci 9:545-556.

Rudebeck PH, Walton ME, Smyth AN, Bannerman DM, Rushworth MF
(2006) Separate neural pathways process different decision costs. Nat Neurosci 9:1161-1168.

Rudebeck PH, Behrens TE, Kennerley SW, Baxter MG, Buckley MJ, Walton ME, Rushworth MF (2008) Frontal cortex subregions play distinct roles in choices between actions and stimuli. J Neurosci 28:13775-13785.

Rushworth MF, Behrens TE, Rudebeck PH, Walton ME (2007) Contrasting roles for cingulate and orbitofrontal cortex in decisions and social behaviour. Trends Cogn Sci 11:168-176.

Schweighofer N, Shishida K, Han CE, Okamoto Y, Tanaka SC, Yamawaki S, Doya K (2006) Humans can adopt optimal discounting strategy under real-time constraints. PLoS Comput Biol 2:e152.

Sescousse G, Redouté, Dreher JC (2010) The architecture of reward value coding in the orbitofrontal cortex. J Neurosci 30:13095-13104.

Shidara M, Richmond BJ (2002) Anterior cingulate: single neuronal signals related to degree of reward expectancy. Science 296:1709-1711.

Smith DV, Hayden BY, Truong TK, Song AW, Platt ML, Huettel SA (2010) Distinct value signals in anterior and posterior ventromedial prefrontal cortex. J Neurosci 30:2490-2495.

Spira A, Bajos N, Giami A, Laurent R, Leridon H (1993) Les comportements sexuels en France. Paris: La Documentation Française.

Stevens JR, Rosati AG, Ross KR, Hauser MD (2005) Will travel for food: spatial discounting in two new world monkeys. Curr Biol 15:1855-1860.

Tanaka SC, Doya K, Okada G, Ueda K, Okamoto Y, Yamawaki S (2004) Prediction of immediate and future rewards differentially recruits cortico-basal ganglia loops. Nat Neurosci 7:887-893.

Walton ME, Bannerman DM, Alterescu K, Rushworth MF (2003) Functional specialization within medial frontal cortex of the anterior cingulate for evaluating effort-related decisions. J Neurosci 23:6475-6479.

Walton ME, Kennerley SW, Bannerman DM, Phillips PE, Rushworth MF (2006) Weighing up the benefits of work: behavioral and neural analyses of effort-related decision making. Neural Netw 19:1302-1314.

Walton ME, Groves J, Jennings KA, Croxson PL, Sharp T, Rushworth MF, Bannerman DM (2009) Comparing the role of the anterior cingulate cortex and 6-hydroxydopamine nucleus accumbens lesions on operant effort-based decision making. Eur J Neurosci 29:1678-1691.

Weiskopf N, Hutton C, Josephs O, Deichmann R (2006) Optimal EPI parameters for reduction of susceptibility-induced BOLD sensitivity losses: a whole-brain analysis at $3 \mathrm{~T}$ and $1.5 \mathrm{~T}$. Neuroimage 33:493-504.

Winstanley CA, Theobald DE, Cardinal RN, Robbins TW (2004) Contrasting roles of basolateral amygdala and orbitofrontal cortex in impulsive choice. J Neurosci 24:4718-4722.

Wittmann M, Leland DS, Paulus MP (2007) Time and decision making: differential contribution of the posterior insular cortex and the striatum during a delay discounting task. Exp Brain Res 179:643-653.

Yacubian J, Gläscher J, Schroeder K, Sommer T, Braus DF, Büchel C (2006) Dissociable systems for gain- and loss-related value predictions and errors of prediction in the human brain. J Neurosci 26:9530-9537. 